\title{
Plasmon-enhanced photocurrent generation and water oxidation from visible to near-infrared wavelengths
}

\author{
Kosei Ueno ${ }^{1,2}$ and Hiroaki Misawa ${ }^{1}$ \\ This paper presents recent investigations of plasmon-enhanced photoelectric conversion and water oxidation by visible and near- \\ infrared light irradiation. Since the discovery of the Honda-Fujishima effect in 1972, significant efforts have been devoted to \\ lengthening the light-energy conversion wavelength. In this context, plasmonic photoelectric conversion has been recently \\ demonstrated at visible-to-near-infrared wavelengths without deteriorating photoelectric conversion by employing titanium \\ dioxide $\left(\mathrm{TiO}_{2}\right)$ single-crystal photoelectrodes, in which gold nanorods are elaborately arrayed on the surface. A potassium \\ perchlorate aqueous solution was employed as an electrolyte solution without additional electron donors; thus, water molecules \\ provided the electrons. The stoichiometric evolution of oxygen and hydrogen peroxide as a result of the four- or two-electron \\ oxidation of water molecules, respectively, was accomplished with near-infrared light irradiation using the plasmonic optical \\ antenna effect. As there is very little overpotential for water oxidation, these results constitute a significant advancement in this \\ field. In addition, this photoelectric conversion system could potentially be employed in artificial photosynthesis systems that \\ exceed the photosynthetic capabilities of plants by allowing for photoconversion over a wide range of wavelengths.
}

NPG Asia Materials (2013) 5, e61; doi:10.1038/am.2013.42; published online 6 September 2013

Keywords: gold nanorod; localized surface plasmon resonance; near-infrared light; photoelectric conversion; plasmonic chemistry; water oxidation

\section{INTRODUCTION}

To realize a future low-carbon society, it is necessary to create systems that can convert solar energy to electrical or chemical energy. A system compatible with the broad spectrum of sunlight, which includes the visible to near-infrared wavelengths, must be developed to achieve efficient solar energy conversion. Although the $\mathrm{p}-\mathrm{n}$ junction silicon solar cell widely used in photovoltaic panels is now commercially available for home use, its photoelectric conversion efficiency is only $\sim 17 \%$, with $30 \%$ marking the upper theoretical limit. ${ }^{1}$ To consider photovoltaics as a future substitute for nuclear power generation, it is necessary to improve solar energy conversion efficiencies. Therefore, the search for efficient light-energy conversion systems based on alternative principles has attracted considerable attention. The solar irradiance spectrum observed on the surface of the Earth is illustrated in Figure 1. Remarkably, only $\sim 4 \%$ of this spectrum constitutes ultraviolet radiation $(<400 \mathrm{~nm})$, whereas visible light $(400-800 \mathrm{~nm})$ comprises $50 \%$ and infrared light $(>800 \mathrm{~nm}$ ) comprises the remaining $46 \%$. Silicon solar cells exhibit a limited ability to conduct photoelectric conversion of infrared light longer than $1000 \mathrm{~nm}$ in wavelength due to the interference of the silicon band gap. Therefore, to develop an efficient solar cell, it is necessary to improve the photoconversion of infrared light, which is a significant component of the solar spectrum.

In 1972, Honda and Fujishima first demonstrated that photoelectric conversion could be accomplished at very high efficiencies in the ultraviolet wavelength region using a titanium dioxide $\left(\mathrm{TiO}_{2}\right)$ semiconductor electrode as a working electrode in photoelectrochemical measurements. ${ }^{2}$ Since then, many attempts to extend photoelectric conversion and photocatalytic reaction to longer wavelengths have been made. ${ }^{3-6}$ Solar cells effective over a broad wavelength range, such as tandem solar cells employing semiconductors that exhibit different band gaps 7,8 and dyesensitized solar cells, which extend wavelength efficiency using organic dyes with various absorption bands, have previously been developed at the laboratory level. ${ }^{9-11}$ However, these systems require the use of two or more different materials and molecules, thus complicating their fabrication. In contrast, metallic nanostructures exhibiting localized surface plasmon resonance (LSPR) can be made to exhibit varying resonant wavelengths by tailoring the nanoparticle structural parameters, such as size, shape and spatial arrangement in an array. ${ }^{12-14}$ Therefore, it is possible to control the resonant wavelength in a single substance. Moreover, LSPR gives rise to an optical antenna effect, which efficiently harvests light and localizes electromagnetic waves at the nanoscale. ${ }^{15-19}$ Therefore, it is understood that metallic nanostructures act as photoelectric conversion systems that enable the effective utilization of photons, having a role similar to that of chlorophyll in photosynthesis. ${ }^{20-23}$ Although many photoelectric conversion systems using LSPR have been previously reported, the wavelengths at which photoelectric

${ }^{1}$ Research Institute for Electronic Science, Hokkaido University, Sapporo, Japan and 2PRESTO, Japan Science and Technology Agency, Kawaguchi, Japan Correspondence: Professor H Misawa, Research Institute for Electronic Science, Hokkaido University, N21, W10, Kita-ku, Sapporo 001-0021, Japan. E-mail: misawa@es.hokudai.ac.jp

Received 30 May 2013; revised 16 June 2013; accepted 18 June 2013 


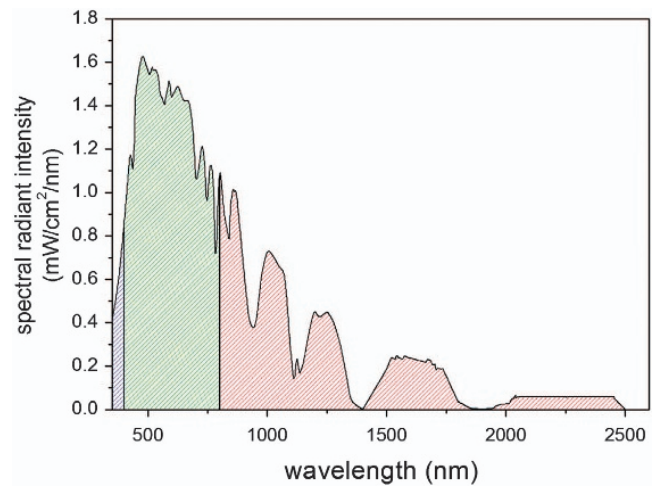

Figure 1 The solar irradiance spectrum observed on the surface of Earth.

conversion occurs did not extend beyond $700 \mathrm{~nm},{ }^{24-31}$ creating a need to explore photoelectric conversion at infrared wavelengths past $800 \mathrm{~nm}$.

Recently, plasmonic photoelectric conversion from visible to nearinfrared wavelengths $(<1300 \mathrm{~nm})$ without deteriorating photoelectric conversion using $\mathrm{TiO}_{2}$ single-crystal photoelectrodes in which gold nanorods (Au NRs) were elaborately arrayed on the surface was demonstrated. ${ }^{32}$ In this review, we describe recent studies concerning the optical antenna effect induced by LSPR, the spectral properties of $\mathrm{Au}$ NRs, and the plasmonic photoelectric conversion behavior at visible to near-infrared wavelengths of light, as well as the experimental approach for investigating the mechanism of plasmonic photoelectric conversion.

\section{OPTICAL ANTENNA EFFECT INDUCED BY LSPR}

Light comprises electromagnetic waves in which the electric field oscillates perpendicularly to the direction of propagation of the wave. When metallic nanoparticles, such as silver and $\mathrm{Au}$, are irradiated by light, a collective motion of the conduction electrons at the metallic nanoparticle surface is induced by the oscillating electric field. ${ }^{13,33}$ As a result, a dipole and quadrupole form because a charge bias arises on the surface of the metallic nanoparticle. ${ }^{13,34-37}$ Furthermore, because an electric field arises as a result of the differential electronic charge, localized electromagnetic waves, also called 'near-field light', form on the metallic particle surface. This near-field light differs from ordinary light (light that can be propagated, also called propagating light), and is localized in the vicinity of the metallic surface. This phenomenon is known as LSPR. The near-field light continues to exist at the nanoparticle surface until the collective motion of the conduction electrons becomes disordered (LSPR phase relaxation). As the phase relaxation time of LSPR is approximately several femtoseconds (depending on the type of metal, size and shape), near-field light will exist at the metal particle surface only for that time. ${ }^{12,38}$ However, propagating light will pass metallic nanoparticles tens of nanometers in size in $<0.1 \mathrm{fs}$, which is shorter than the phase-relaxation time of LSPR by two orders of magnitude. Moreover, because the density of the electric charge is highest at the ends of the metal particles that are aligned with the electric field of the incident light, the electromagnetic field concentrates at the ends of the particles along the incident polarization. ${ }^{39,40}$ This phenomenon results in the temporary spatial confinement of light at the metallic nanoparticle surface as a consequence of the LSPR excitation. ${ }^{22,41}$ Therefore, on the metallic nanoparticle surface, the electromagnetic field is enhanced by roughly two orders of magnitude compared with the electromagnetic field

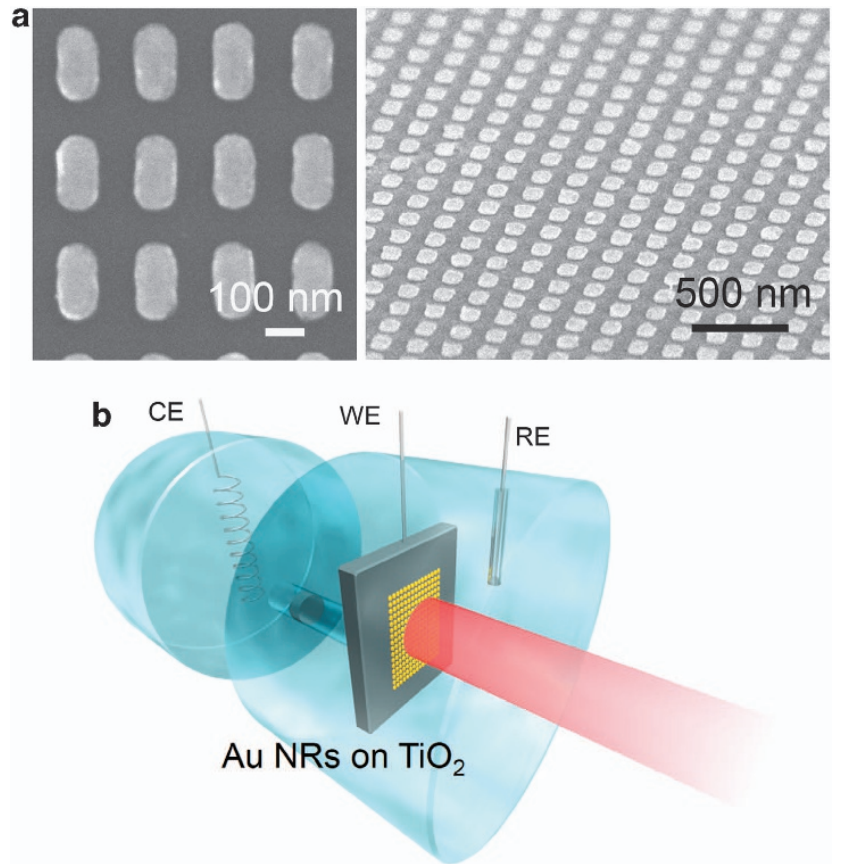

Figure 2 (a) Scanning electron microscope images of $\mathrm{Au}$ NRs on $\mathrm{TiO}_{2}$ (left) Scanning electron microscope image of the same substrate tilted by $75^{\circ}$ (right). (b) A schematic illustration of the photoelectrochemical cell.

intensity of the incident light. It therefore becomes possible to harvest photons in a manner similar to a metallic antenna and to localize them to a nanometer-sized spatial region. As the light-matter coupling process is enhanced by this electromagnetic field enhancement effect, the enhancement of fluorescence, ${ }^{42}$ Raman scattering signals $^{43}$ and other phenomena have been reported. On the basis of the electromagnetic field enhancement effect, there is a possibility that metallic nanostructures exhibiting LSPR function as an antenna similar to the reaction-center chlorophyll molecules of plants, resulting in an enhancement in the photoelectric conversion efficiency of solar cells and allowing for application in artificial photosynthesis systems.

\section{PLASMONIC PHOTOELECTRIC CONVERSION BY VISIBLE AND NEAR-INFRARED LIGHT}

The Au NRs used in the study by Nishijima et al. ${ }^{32}$ were fabricated in the range of $2.5 \mathrm{~mm}$ around on a rutile-type single-crystal $\mathrm{TiO}_{2}$ substrate $(0.05 \mathrm{wt} \% \mathrm{Nb}$-doped) using electron beam lithography and the lift-off method to create optical antenna structures. Figure $2 \mathrm{a}$ depicts the scanning electron microscope images of the Au NRs (each $100 \times 200 \times 30 \mathrm{~nm})$ on $\mathrm{TiO}_{2}$. To measure the photoelectric conversion properties of the photoelectrode, a conventional three-electrode photoelectrochemical measurement system was employed. A schematic illustration of the photoelectrochemical cell is displayed in Figure 2b. In this measurement system, a $\mathrm{TiO}_{2}$ substrate loaded with Au NPs was used as the working electrode, whereas a platinum wire and saturated calomel electrode were used as the counter electrode and reference electrode, respectively. In the photoelectrochemical measurement system, the working electrode was covered with a Teflon-made plate having a window $(\varphi: 2 \mathrm{~mm})$ through which light can irradiate only the Au NRs fabricated on the working electrode. Significantly, only a potassium perchlorate aqueous 
solution $\left(0.1 \mathrm{~mol} \mathrm{dm}^{-3}\right)$ was used as the electrolyte solution without the addition of other electron donors.

Figure 3a displays the extinction spectra of the Au NRs in the electrolyte solution under nonpolarized and polarized conditions. Two distinct LSPR bands were clearly observed near 650 and $1000 \mathrm{~nm}$ under the nonpolarized conditions (black curve), in agreement with the extinction spectra of chemically synthesized Au NRs. ${ }^{44,45}$ The measured spectra in Figure $3 \mathrm{a}$ indicate that the transverse mode (T-mode, $\lambda_{\text {max }}: 650 \mathrm{~nm}$; the spectrum shown in blue) and longitudinal mode (L-mode, $\lambda_{\text {max }}: 1000 \mathrm{~nm}$; the spectrum shown in red) that are perpendicular and parallel, respectively, to the $\mathrm{Au} \mathrm{NR}$ arrays can be selectively excited by controlling the polarization of the incident light. ${ }^{46}$ Figure $3 \mathrm{~b}$ displays a linear sweep voltammogram under irradiation at wavelengths ranging from 500 to $1300 \mathrm{~nm}$ with and without Au NRs. Importantly, no photocurrent was observed from the $\mathrm{TiO}_{2}$ single-crystal photoelectrode in the absence of $\mathrm{Au}$ NRs under irradiation with visible and nearinfrared light. However, an anodic photocurrent was clearly observed at a positive potential above $-0.3 \mathrm{~V}$ (vs saturated calomel electrode) using the Au NR-loaded $\mathrm{TiO}_{2}$ photoelectrode. Figure $3 \mathrm{c}$ displays the action spectra of the incident photon-to-current conversion efficiency (IPCE) under nonpolarized and polarized conditions at an applied potential of $+0.3 \mathrm{~V}$ (vs saturated calomel electrode). The shapes and peak wavelengths of the IPCE action spectra were in close agreement with those of the plasmon resonance bands with high reproducibility. According to the measurement of the IPCE action spectrum under linearly polarization conditions, the IPCE values of the photocurrent were 6.3 and $8.4 \%$, corresponding to the LSPR spectra in the T-mode at $650 \mathrm{~nm}$ and the L-mode at $1000 \mathrm{~nm}$, respectively. These observations suggested that the electron injection from the Au NRs to the $\mathrm{TiO}_{2}$ single-crystal substrate is induced by LSPR excitation.

\section{TEMPERATURE DEPENDENCE OF THE PLASMONIC PHOTOELECTRIC CONVERSION}

The effect of temperature on the plasmonic photoelectric conversion was explored at the plasmon wavelengths of $650 \mathrm{~nm}$ (T-mode) and $1000 \mathrm{~nm}$ (L-mode) and at the $450 \mathrm{~nm}$ wavelength that can induce the interband transition of $\mathrm{Au}$ from the d-bands to the sp-conduction band. ${ }^{47,48}$ As illustrated in the current-potential curves measured at various temperatures in Figure $4 \mathrm{a}$, it is clear that the reduction of the photocurrent that accompanies increases in temperature in silicon solar cells was not observed in the plasmonic photoelectrode; rather, the photocurrent was observed to increase with increasing temperature. Using the temperature dependence of the photocurrent, an Arrhenius plot for each irradiation wavelength was created in Figure $4 \mathrm{~b}$. It was determined that $\sim 12 \mathrm{~kJ} \mathrm{~mol}^{-1}$ of activation energy exists in the LSPR excitation (650 and $1000 \mathrm{~nm}$ wavelengths), whereas the $\mathrm{Au}$ interband transition (450 nm wavelength) contains $29 \mathrm{~kJ} \mathrm{~mol}^{-1}$ of energy. This difference in energy implies that the process of electron transfer may differ between the interband transition and LSPR excitation. Furthermore, the dependence of the plasmonic photoelectric conversion on the irradiation time for $200 \mathrm{~h}$ was measured. It was confirmed that a stable photocurrent can persist for $200 \mathrm{~h}$ in aqueous electrolyte solutions lacking electron donor molecules, as revealed in Figure 4c. This result suggests that water molecules may serve as an electron source. Specifically, it was hypothesized that oxygen $\left(\mathrm{O}_{2}\right)$ or hydrogen peroxide $\left(\mathrm{H}_{2} \mathrm{O}_{2}\right)$ was evolved as a result of water oxidation at the Au NR-loaded $\mathrm{TiO}_{2}$ photoelectrode.

\section{DETERMINING THE ELECTRON SOURCE IN THE PLASMONIC PHOTOELECTRIC CONVERSION SYSTEM}

On the basis of the abovementioned observations, the source of electrons in the plasmonic photoelectric conversion system was
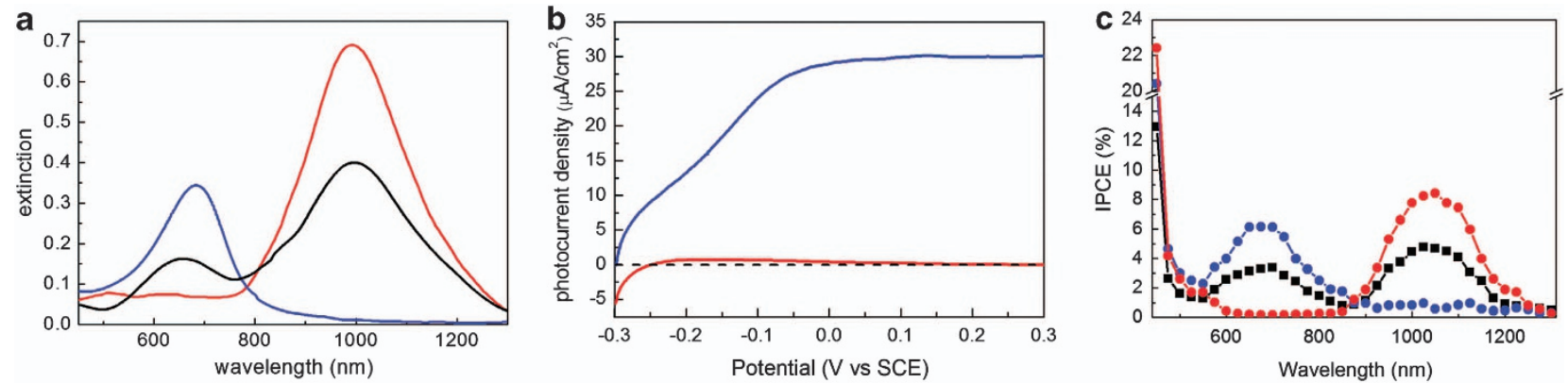

Figure 3 (a) Extinction spectra of the Au NRs in water. Black: nonpolarized light. Red and blue: L- and T-modes, respectively, under irradiation with linearly polarized light. (b) Linear sweep voltammograms under irradiation with light in the wavelength range from 500 to $1300 \mathrm{~nm}$ with (blue) and without (red) Au NRs. The sweep rate was $5 \mathrm{mVs}^{-1}$ (c) IPCE action spectra. Black: nonpolarized light, Red: L-mode and Blue: T-mode.
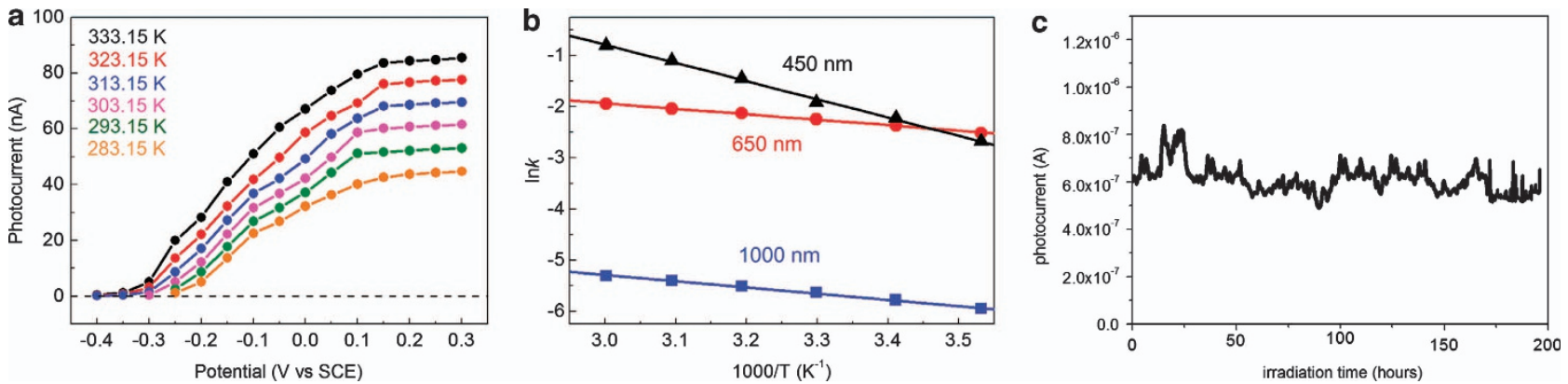

Figure 4 (a) Temperature dependence of the current-potential curves. (b) Arrhenius plot (applied potential: 0.3V vs saturated calomel electrode). (c) Irradiation time dependence of the photocurrent value over $200 \mathrm{~h}$. 
determined. ${ }^{49}$ The amount of $\mathrm{O}_{2}$ and $\mathrm{H}_{2} \mathrm{O}_{2}$ that evolved from the oxidation of water was quantitatively determined by gas chromatography/mass spectrometry (GC-MS) using water-(18-O) $(10 \%$ isotopic purity $)^{50,51}$ and by absorptiometry using oxo(5,10,15, 20tetra(4-pyridyl)porphinato)titanium(IV) as an $\mathrm{H}_{2} \mathrm{O}_{2}$ indicator. ${ }^{52}$ To analyze the quantity of $\mathrm{O}_{2}$ and $\mathrm{H}_{2} \mathrm{O}_{2}$ that evolved for an observed photocurrent, $\mathrm{O}_{2}, \mathrm{H}_{2} \mathrm{O}_{2}$ and the number of electrons were quantitatively measured to assess the relationship between the irradiation time and photocurrent. Figure 5 displays the reaction yield of $\mathrm{O}_{2}$ and $\mathrm{H}_{2} \mathrm{O}_{2}$ as a function of the observed photocurrent and its corresponding IPCE action spectrum. At the irradiation wavelengths of 500 and $600 \mathrm{~nm}$, which can induce the interband transition of $\mathrm{Au}$ and the LSPR T-mode, a nearly stoichiometric evolution of oxygen was clearly achieved. However, at the irradiation wavelengths of 800 and $1000 \mathrm{~nm}$, which can induce the LSPR L-mode, the amount of evolved $\mathrm{O}_{2}$ decreased and $\mathrm{H}_{2} \mathrm{O}_{2}$ was generated instead. These results indicate that water molecules served as the electron donor in the plasmonic photoelectric conversion system and that a nearly stoichiometric evolution of $\mathrm{O}_{2}$ and $\mathrm{H}_{2} \mathrm{O}_{2}$ was achieved as a result of the oxidation of a water molecule with four or two photogenerated holes, respectively. Although numerous studies have achieved water oxidation or water splitting using the irradiation of visible light below wavelengths of $700 \mathrm{~nm},{ }^{31,53-57}$ this study is the first to achieve the stoichiometric evolution of $\mathrm{O}_{2}$ from water molecules with the irradiation of near-infrared light at a wavelength of $1000 \mathrm{~nm}$ $(1.24 \mathrm{eV})$. This result means that water oxidation via a four-electron transfer was accomplished by using a plasmonic photoelectric conversion system. If the method of electron transfer at the electrode interface is induced by LSPR, an interface between different phases that dramatically reduces energy loss can be designed. It is expected that the ultimate solar energy conversion system can convert $\sim 80 \%(<1300 \mathrm{~nm})$ of the light energy in sunlight to chemical energy.

\section{MECHANISM OF PHOTOELECTRIC CONVERSION}

The current section elaborates on the mechanism of the plasmonic photoelectric conversion. As illustrated in the energy diagram in Figure 6, it is hypothesized that an excited electron is transferred to the conduction band of $\mathrm{TiO}_{2}$ immediately following the inter- or intraband transition of the Au nanoparticles induced by the plasmonically enhanced optical near-field, ${ }^{58}$ thus leaving a hole trapped to the surface states of the $\mathrm{TiO}_{2}$ near the $\mathrm{Au} / \mathrm{TiO}_{2} /$ water interface; the trapped holes can subsequently induce the oxidation of water molecules. ${ }^{59}$ It is possible for multiple holes to form and become trapped at the restricted nanospace near the $\mathrm{Au} / \mathrm{TiO}_{2}$ interface because the plasmonically enhanced optical near-field is induced at both ends of the $\mathrm{Au}$ nanoparticles, especially at the $\mathrm{Au} / \mathrm{TiO}_{2} /$ water interface, as determined using electromagnetic simulations. Therefore, the overpotential can be ignored because the four trapped holes can react with two water molecules simultaneously. Furthermore, there is a possibility that the activation energy at the plasmon resonance wavelengths is lower than that at the wavelength that induces the interband transition of $\mathrm{Au}$, because the direct excitation of $\mathrm{Au}$ can occur throughout the Au NRs. On the basis of the proposed mechanism, the plasmonically enhanced optical near-field would promote water oxidation, resulting in the evolution of $\mathrm{O}_{2}$ and $\mathrm{H}_{2} \mathrm{O}_{2}$ even at low-energy wavelengths in the near-infrared region. However, whether the excitation process involves an interband or intraband transition in the $\mathrm{Au}$, a hot electron transfer, or the direct excitation of electrons trapped in the surface states of the $\mathrm{TiO}_{2}$ remains unknown. To understand this mechanism in greater detail,

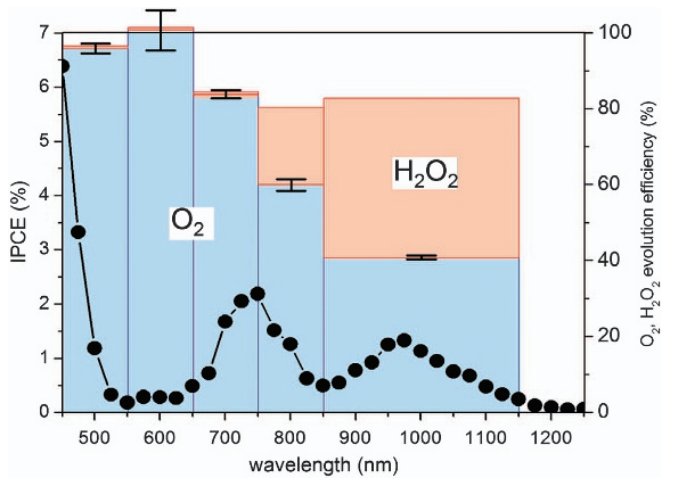

Figure 5 Reaction yield of $\mathrm{O}_{2}$ and $\mathrm{H}_{2} \mathrm{O}_{2}$ as a function of the observed photocurrent and its corresponding IPCE action spectrum.

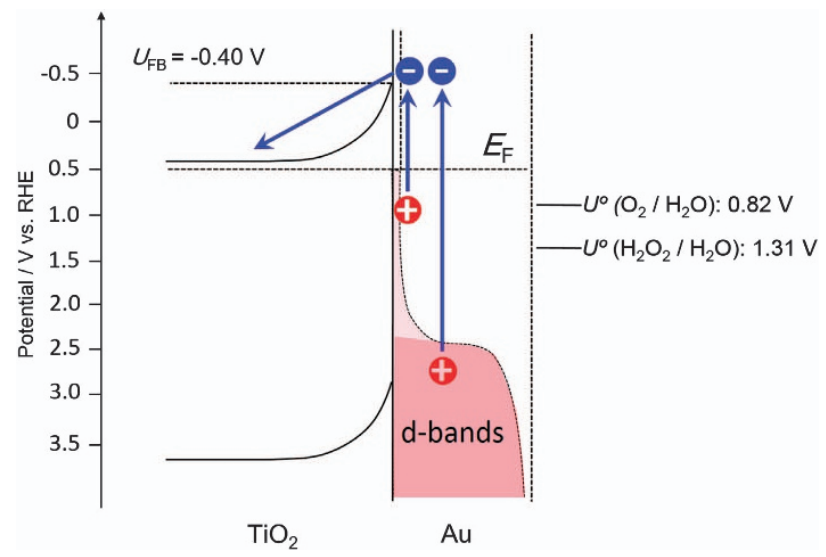

Figure 6 Energy diagram of the Au NR-loaded $\mathrm{TiO}_{2}$ photoelectrode system. $U_{\mathrm{FB}}$ and $U_{0}$ exhibit the flat band potential of $\mathrm{TiO}_{2}$ and the redox potentials versus reversible hydrogen electrode, respectively. The symbols [-] and [+] indicate the electron and the hole, respectively.

it is important to study the electron-transfer dynamics using timeresolved photoemission electron microscopy and transient absorption measurements pumped by an ultra-short pulse laser, as well to characterize the multiple holes trapped at the $\mathrm{TiO}_{2}$ surface states near the $\mathrm{Au} / \mathrm{TiO}_{2} /$ water interface using atomic-resolution scanning transmission electron microscopy and electron energy loss spectroscopy under light irradiation conditions.

\section{CONCLUSION}

In this review, we summarized the recent results regarding plasmoninduced photoelectric conversion and water oxidation using the irradiation of visible and near-infrared light. Although $\mathrm{TiO}_{2}$ was not directly excited in this study, the Honda-Fujishima effect was observed even with the irradiation of near-infrared light by harnessing the plasmonic antenna effect. In particular, water oxidation via a fourelectron transfer with near-infrared radiation would be challenging owing to a large overpotential. It was speculated that multiple holes formed at the restricted nanospace as a result of the electromagnetic field enhancement induced by LSPR excitation, enabling the oxidation of two water molecules simultaneously and dramatically reducing the overpotential. These results indicate that this photoelectric conversion system can be applied to artificial photosynthesis systems. Significantly, this system constitutes an enormous stride forward by 
providing the potential to exceed the photosynthetic capabilities of green plants through photosystem II $(\lambda: 680 \mathrm{~nm})$ by enabling the conversion of a wider range of wavelengths, including near-infrared light, which is not currently used in conventional artificial photosynthesis systems.

\section{ACKNOWLEDGEMENTS}

We are extremely grateful to Dr Yoshiaki Nishijima (present affiliation: Yokohama National University), Dr Yukie Yokota (present affiliation: RIKEN) and Mr Yuki Kotake at Hokkaido University for their experimental contributions, as well as Professor Kei Murakoshi at Hokkaido University and Professor Haruo Inoue at Tokyo Metropolitan University for their fruitful discussions. This study was supported by funding from the Ministry of Education, Culture, Sports, Science, and Technology of Japan: KAKENHI Grant-in-Aid for Scientific Research no. 23225006, Nanotechnology Platform (Hokkaido University) and the Low-Carbon Research Network of Japan.

1 Shockley, W. \& Queisser, H. J. Detailed balance limit of efficiency of p-n junction solar cells. J. Appl. Phys. 32, 510-519 (1961).

2 Fujishima, A. \& Honda, K. Electrochemical photolysis of water at a semiconductor electrode. Nature 238, 37-38 (1972).

3 Asahi, R., Morikawa, T., Ohwaki, T., Aoki, K. \& Taga, Y. Visible-light photocatalysis in nitrogen-doped titanium oxides. Science 293, 269-271 (2001).

4 Yu, J. C., Yu, J. G., Ho, W. K., Jiang, Z. T. \& Zhang, L. Z. Effects of F- doping on the photocatalytic activity and microstructures of nanocrystalline $\mathrm{TiO}_{2}$ powders. Chem. Mater. 14, 3808-3816 (2002).

5 Ohno, T., Akiyoshi, M., Umebayashi, T., Asai, K., Mitsui, T. \& Matsumura, M. Preparation of S-doped $\mathrm{TiO}_{2}$ photocatalysts and their photocatalytic activities under visible light. Appl. Catal. A 265, 115-121 (2004).

6 Irie, H., Miura, S., Kamiya, K. \& Hashimoto, K. Efficient visible light-sensitive photocatalysts: grafting $\mathrm{Cu}(\mathrm{II})$ ions onto $\mathrm{TiO}_{2}$ and $\mathrm{WO}_{3}$ photocatalysts. Chem. Phys. Lett. 457, 202-205 (2008).

7 Luque, A. \& Marti, A. Increasing the efficiency of ideal solar cells by photon induced transitions at intermediate levels. Phys. Rev. Lett. 78, 5014-5017 (1997).

8 Hanna, M. C. \& Nozik, A. J. Solar conversion efficiency of photovoltaic and photoelectrolysis cells with carrier multiplication absorbers. J. Appl. Phys. 100, 074510 (2006)

9 Oregan, B. \& Gratzel, M. A low-cost, high-efficiency solar-cell based on dye-sensitized colloidal $\mathrm{TiO}_{2}$ films. Nature 353, 737-740 (1991).

10 Bach, U., Lupo, D., Comte, P., Moser, J. E., Weissortel, F., Salbeck, J., Spreitzer, H. \& Gratzel, M. Solid-state dye-sensitized mesoporous $\mathrm{TiO}_{2}$ solar cells with high photon-toelectron conversion efficiencies. Nature 395, 583-585 (1998).

11 Nazeeruddin, M. K., Kay, A., Rodicio, I., Humphrybaker, R., Muller, E., Liska, P., Vlachopoulos, N. \& Gratzel, M. Conversion of light to electricity by cis-X2bis(2,2'bipyridyl-4,4'-dicarboxylate)ruthenium(II) charge-transfer sensitizers ( $\mathrm{X}=\mathrm{Cl}-, \mathrm{Br}-, \mathrm{I}-$, $\mathrm{CN}-$, and $\mathrm{SCN}-$ ) on nanocrystalline $\mathrm{TiO}_{2}$ electrodes. J. Am. Chem. Soc. 115, 6382-6390 (1993).

12 Link, S. \& El-Sayed, M. A. Spectral properties and relaxation dynamics of surface plasmon electronic oscillations in gold and silver nanodots and nanorods. J. Phys. Chem. B 103, 8410-8426 (1999).

13 Kelly, K. L., Coronado, E., Zhao, L. L. \& Schatz, G. C. The optical properties of metal nanoparticles: the influence of size, shape, and dielectric environment. J. Phys. Chem. B 107, 668-677 (2003)

14 Yokota, Y., Ueno, K., Mizeikis, V., Juodkazis, S., Sasaki, K. \& Misawa, H. Optical characterization of plasmonic metallic nanostructures fabricated by high-resolution lithography. J. Nanophoton 1, 011594 (2007).

15 Xu, H. X., Aizpurua, J., Kall, M. \& Apell, P. Electromagnetic contributions to singlemolecule sensitivity in surface-enhanced Raman scattering. Phys. Rev. E 62, 43184324 (2000).

16 Hao, E. \& Schatz, G. C. Electromagnetic fields around silver nanoparticles and dimers. J. Chem. Phys. 120, 357-366 (2004).

17 Sawai, Y., Takimoto, B., Nabika, H., Ajito, K. \& Murakoshi, K. Observation of a small number of molecules at a metal nanogap arrayed on a solid surface using surfaceenhanced Raman scattering. J. Am. Chem. Soc. 129, 1658-1662 (2007).

18 Ueno, K., Juodkazis, S., Shibuya, T., Yokota, Y., Mizeikis, V., Sasaki, K. \& Misawa, H. Nanoparticle plasmon-assisted two-photon polymerization induced by incoherent excitation source. J. Am. Chem. Soc. 130, 6928-6929 (2008).

19 Ueno, K., Takabatake, S., Nishijima, Y., Mizeikis, V., Yokota, Y. \& Misawa, H. Nanogapassisted surface plasmon nanolithography. J. Phys. Chem. Lett. 1, 657-662 (2010).

20 Muhlschlegel, P., Eisler, H. J., Martin, O. J. F., Hecht, B. \& Pohl, D. W. Resonant optical antennas. Science 308, 1607-1609 (2005).

21 Sundaramurthy, A., Schuck, P. J., Conley, N. R., Fromm, D. P., Kino, G. S. \& Moerner, W. E. Toward nanometer-scale optical photolithography: Utilizing the near-field of bowtie optical nanoantennas. Nano Lett. 6, 355-360 (2006).
22 Gao, S. Y., Ueno, K. \& Misawa, H. Plasmonic antenna effects on photochemical reactions. Acc. Chem. Res. 44, 251-260 (2011).

23 Knight, M. W., Sobhani, H., Nordlander, P. \& Halas, N. J. Photodetection with active optical antennas. Science 332, 702-704 (2011).

24 Tian, Y. \& Tatsuma, T. Plasmon-induced photoelectrochemistry at metal nanoparticles supported on nanoporous $\mathrm{TiO}_{2}$. Chem. Commun. 1810-1811 (2004).

25 Tian, Y. \& Tatsuma, T. Mechanisms and applications of plasmon-induced charge separation at $\mathrm{TiO}_{2}$ films loaded with gold nanoparticles. J. Am. Chem. Soc. 127, $7632-7637$ (2005).

26 Yu, K. F., Tian, Y. \& Tatsuma, T. Size effects of gold nanaoparticles on plasmon-induced photocurrents of gold- $\mathrm{TiO}_{2}$ nanocomposites. Phys. Chem. Chem. Phys. 8, 5417-5420 (2006).

27 Wang, P., Xie, T. F., Li, H. Y., Peng, L. A., Zhang, Y., Wu, T. S., Pang, S., Zhao, Y. F. \& Wanga, D. J. Synthesis and plasmon-induced charge-transfer properties of monodisperse gold-doped titania microspheres. Chem. Eur. J 15, 4366-4372 (2009).

28 Lee, Y. K., Jung, C. H., Park, J., Seo, H., Somorjai, G. A. \& Park, J. Y. Surface plasmondriven hot electron flow probed with metal-semiconductor nanodiodes. Nano Lett. 11, 4251-4255 (2011).

29 Liu, J. \& Chen, F. Y. Plasmon enhanced photoelectrochemical activity of Ag-Cu nanoparticles on $\mathrm{TiO}_{2} / \mathrm{Ti}$ Substrates. Int. J. Electrochem. Sci. 7, 9560-9572 (2012).

30 Hashimoto, T., Fukunishi, Y., Zheng, B., Uraoka, Y., Hosoi, T., Shimura, T. \& Watanabe, $\mathrm{H}$. Electrical detection of surface plasmon resonance phenomena by a photoelectronic device integrated with gold nanoparticle plasmon antenna. Appl. Phys. Lett. 102, 083702 (2013).

31 Shi, X., Ueno, K., Takabayashi, N. \& Misawa, H. Plasmon-enhanced photocurrent generation and water oxidation with a gold nanoisland-loaded titanium dioxide photoelectrode. J. Phys. Chem. C 117, 2494-2499 (2013).

32 Nishijima, Y., Ueno, K., Yokota, Y., Murakoshi, K. \& Misawa, H. Plasmon-assisted photocurrent generation from visible to near-infrared wavelength using a Au-nanorods/ $\mathrm{TiO}_{2}$ electrode. J. Phys. Chem. Lett. 1, 2031-2036 (2010).

33 Wang, D. W. \& Das Sarma, S. Resonant Raman scattering by charge-density and singleparticle excitations in semiconductor nanostructures: a generalized interband-resonant random-phase-approximation theory. Phys. Rev. B 65, 125322 (2002).

34 Jin, R. C., Cao, Y. W., Mirkin, C. A., Kelly, K. L., Schatz, G. C. \& Zheng, J. G. Photoinduced conversion of silver nanospheres to nanoprisms. Science 294, 1901-1903 (2001).

35 Krenn, J. R., Schider, G., Rechberger, W., Lamprecht, B., Leitner, A., Aussenegg, F. R. \& Weeber, J. C. Design of multipolar plasmon excitations in silver nanoparticles. Appl. Phys. Lett. 77, 3379-3381 (2000).

36 Imura, K., Nagahara, T. \& Okamoto, H. Near-field two-photon-induced photoluminescence from single gold nanorods and imaging of plasmon modes. J. Phys. Chem. B 109, 13214-13220 (2005)

37 Ueno, K., Takabatake, S., Onishi, K., Itoh, H., Nishijima, Y. \& Misawa, H. Homogeneous nano-patterning using plasmon-assisted photolithography. Appl. Phys. Lett. 99, 011107 (2011).

38 Sonnichsen, C., Franzl, T., Wilk, T., von Plessen, G., Feldmann, J., Wilson, O. \& Mulvaney, P. Drastic reduction of plasmon damping in gold nanorods. Phys. Rev. Lett. 88, 077402 (2002).

39 Hubert, C., Rumyantseva, A., Lerondel, G., Grand, J., Kostcheev, S., Billot, L., Vial, A., Bachelot, R., Royer, P., Chang, S. H., Gray, S. K., Wiederrecht, G. P. \& Schatz, G. C. Near-field photochemical imaging of noble metal nanostructures. Nano Lett. 5, 615-619 (2005).

40 Ueno, K., Juodkazis, S., Shibuya, T., Mizeikis, V., Yokota, Y. \& Misawa, H. Nanoparticle-enhanced photopolymerization. J. Phys. Chem. C 113, 11720-11724 (2009).

41 Ueno, K. \& Misawa, H. Spectral properties and electromagnetic field enhancement effects on nano-engineered metallic nanoparticles. Phys. Chem. Chem. Phys. 15, 4093-4099 (2013).

42 Brolo, A. G., Kwok, S. C., Moffitt, M. G., Gordon, R., Riordon, J. \& Kavanagh, K. L. Enhanced fluorescence from arrays of nanoholes in a gold film. J. Am. Chem. Soc. 127, 14936-14941 (2005).

43 Jeanmaire, D. L. \& Vanduyne, R. P. Surface Raman spectroelectrochemistry.1. heterocyclic, aromatic, and aliphatic-amines adsorbed on anodized silver electrode. J. Electroanal. Chem. 84, 1-20 (1977).

44 Yu, Y. Y., Chang, S. S., Lee, C. L. \& Wang, C. R. C. Gold nanorods: electrochemical synthesis and optical properties. J. Phys. Chem. B 101, 6661-6664 (1997)

$45 \mathrm{Kim}$, F., Song, J. H. \& Yang, P. D. Photochemical synthesis of gold nanorods. J. Am. Chem. Soc. 124, 14316-14317 (2002).

46 Ueno, K., Mizeikis, V., Juodkazis, S., Sasaki, K. \& Misawa, H. Optical properties of nanoengineered gold blocks. Opt. Lett. 30, 2158-2160 (2005).

47 Johnson, P. B. \& Christy, R. W. Optical constants of noble metals. Phys. Rev. B 6, 4370-4379 (1972).

48 Christensen, N. E. High-energy band-structure of gold. Phys. Rev. B 13, 2698-2701 (1976).

49 Nishijima, Y., Ueno, K., Kotake, Y., Murakoshi, K., Inoue, H. \& Misawa, H. Nearinfrared plasmon-assisted water oxidation. J. Phys. Chem. Lett. 3, 1248-1252 (2012).

50 Cohn, M. \& Urey, H. C. Oxygen exchange reactions of organic compounds and water. J. Am. Ceram. Soc. 60, 679-687 (1938).

51 Sharp, Z. D., Atudorei, V. \& Durakiewicz, T. A rapid method for determination of hydrogen and oxygen isotope ratios from water and hydrous minerals. Chem. Geol. 178, 197-210 (2001). 
52 Matsubara, C. \& Takamura, K. Spectrophotometric determination of traces of $\mathrm{H}_{2} \mathrm{O}_{2}$ by the $\mathrm{TI}(\mathrm{IV})-\mathrm{PAR}$ and $\mathrm{V}(\mathrm{V})-\mathrm{XO}$ reagents - application to the assay for glucose, urate and cholesterol in serum. J. Pharmacobiodyn. 3, S3 (1980).

53 Liu, Z. W., Hou, W. B., Pavaskar, P., Aykol, M. \& Cronin, S. B. Plasmon resonant enhancement of photocatalytic water splitting under visible illumination. Nano Lett. 11, 1111-1116 (2011).

54 Ingram, D. B. \& Linic, S. Water splitting on composite plasmonic-metal/semiconductor photoelectrodes: evidence for selective plasmon-induced formation of charge carriers near the semiconductor surface. J. Am. Chem. Soc. 133, 5202-5205 (2011).

55 Primo, A., Marino, T., Corma, A., Molinari, R. \& Garcia, H. Efficient visible-light photocatalytic water splitting by minute amounts of gold supported on nanoparticulate $\mathrm{CeO}_{2}$ obtained by a biopolymer templating method. J. Am. Chem. Soc. 133, 69306933 (2011).

56 Chen, H. M., Chen, C. K., Chen, C.-J., Cheng, L.-C., Wu, P. C., Cheng, B. H., Ho, Y. Z., Tseng, M. L., Hsu, Y.-Y., Chan, T.-S., Lee, J.-F., Liu, R.-S. \& Tsai, D. P. Plasmon inducing effects for enhanced photoelectrochemical water splitting: X-ray absorption approach to electronic structures. ACS Nano 6, 7362-7372 (2012).
57 Kung, W. T., Pai, Y. H., Hsu, Y. K., Lin, C. H. \& Wang, C. M. Surface plasmon assisted $\mathrm{Cu}_{x} \mathrm{O}$ photocatalyst for pure water splitting. Opt. Express 21, A221-A228 (2013).

58 Furube, A., Du, L., Hara, K., Katoh, R. \& Tachiya, M. Ultrafast plasmon-induced electron transfer from gold nanodots into $\mathrm{TiO}_{2}$ nanoparticles. J. Am. Chem. Soc. 129 14852-14853 (2007).

59 Micic, O. I., Zhang, Y. N., Cromack, K. R., Trifunac, A. D. \& Thurnauer, M. C. Trapped holes on $\mathrm{TiO}_{2}$ colloids studied by electron-paramagnetic-resonance. J. Phys. Chem. 97, 7277-7283 (1993).

(c) (i) (2) This work is licensed under a Creative Commons Attribution-NonCommercial-ShareAlike 3.0 Unported License. To view a copy of this license, visit http://creativecommons. org/licenses/by-nc-sa/3.0/

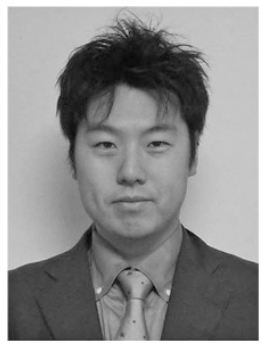

Kosei Ueno is an associate professor at the Research Institute for Electronic Science at Hokkaido University, Japan. He received his PhD in chemistry from Hokkaido University in 2004. From 2004 to 2006, he worked in Professor Hiroaki Misawa's laboratory as a JSPS research fellow. He became an assistant professor at Hokkaido University in 2006 and was promoted to associate professor in 2008. Since 2007, he has held an additional post as a PRESTO researcher at the Japan Science and Technology Agency (JST). His current scientific interests are the fabrication of gold nanostructures defined with sub-nanometer precision and their plasmonic applications, especially in the research field of chemistry.

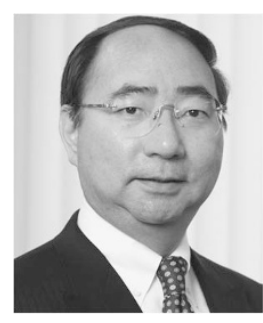

Hiroaki Misawa is a director and professor at the Research Institute for Electronic Science at Hokkaido University, Japan. He received his $\mathrm{PhD}$ in chemistry from the University of Tsukuba in 1984. After postdoctoral positions at the University of Texas (USA) and the University of Tsukuba (Japan) and an assistant professorship at Tsukuba University, he joined Masuhara Microphotoconversion project (ERATO) of the Japan Science and Technology Agency (JST). He became an associate professor in the Department of Engineering at the University of Tokushima in 1993 and was promoted to full professor in 1995. He moved to Hokkaido University as full professor in 2003. His current research interests include photochemistry, light-matter interaction, ultrafast processes in materials, photonic crystals and plasmonic chemistry. 